\title{
The Black Hole
}

\author{
Parashu Ram Poudel \\ Department of Physics, Prithvi Narayan Campus, Pokhara,Nepal \\ Correspondence: parashupoudel@hotmail.com
}

\begin{abstract}
Black holes are the cold remnants of former stars. They are so dense that no matter-not even light-is able to escape their powerful gravitational pull. These are interesting objects from a fundamental physics point of view and play an important role in astrophysics. The knowledge of phenomenon of origin of black hole may lead to new understanding of the nature of the real universe.
\end{abstract}

Keywords: event horizon, schwarzschild radius, accretion disk, white hole, energetic jet, quasars.

\section{Introduction}

Black hole is a region of space-time from which nothing, not even light can escape. A black hole is formed when a star of sufficient mass undergoes gravitational collapse, with most or all of its mass compressed into a sufficiently small area of space, causing infinite spacetime curvature at that point called singularity. Such a massive space-time curvature allows nothing, not even light, escaping from a surface. This is because of distortion of space time caused by extremely compact mass. There is unnoticeable surface called event horizon around a black hole that marks the no return point. The region is referred as black because the region absorbs all the light that falls on it. It cannot reflect anything, just like a completely black body in thermodynamics.

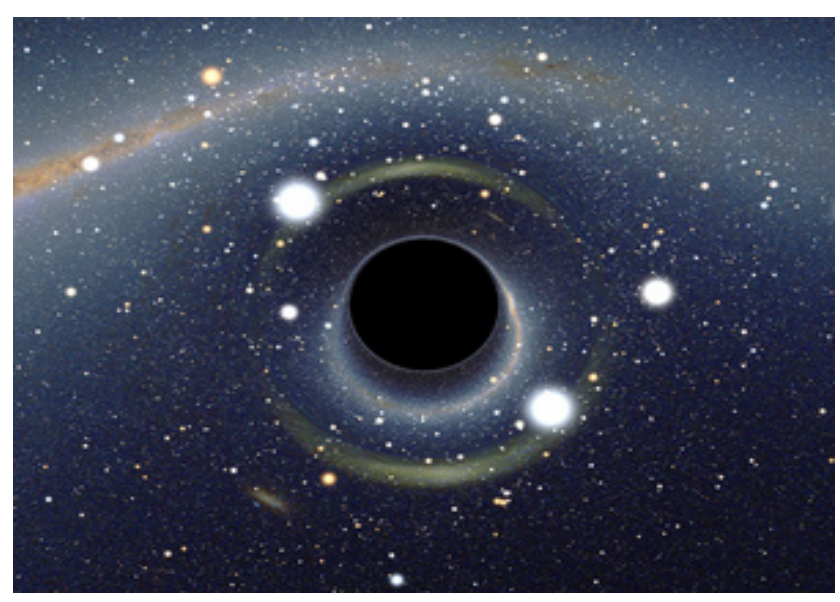

Fig. 1: Black Hole

\section{Historical Background}

Sir Isaac Newton in 1687 described the concept of gravity in his publication Principia. The idea of a body so massive that even light could not escape was first put forward by geologist John Michell in a letter written to Henry Cavendish in 1783 of the Society. In 1796, mathematician Pierre-Simon Laplace promoted the same idea in the first and second editions of his book Exposition du système du Monde Such "dark stars" were largely ignored in the nineteenth century, since it was not understood how a massless wave such as light could be influenced by gravity.

In 1915, Albert Einstein developed his theory of general relativity, having earlier shown that gravity does influence light's motion. Only a few months later In 1916, the German astronomer Karl Schwarzschild attempted to theorize how a star could shrink to become what he called a "Black Hole". Schwarzschild predicted that our sun would have to shrink to less than two miles in radius. He also predicted that even though the sun had shrunk its mass and weight would remain the same, which means that the planets would continue on their orbits, unaffected. Schwarzschild still questioned if stars could become this compact. In 1931, Subrahmanyan Chandrasekhar calculated, using 
special relativity, that a non-rotating body of electrondegenerate matter above a certain limiting mass (now called the Chandrasekhar limit at 1.4 solar masses) has no stable solutions In 1934 W. Baade and F. Zwicky predicted that the collapse of a star, strips the atoms of their electrons, thus making it a Neutron Star. He also predicted that these are only 10-15 miles in diameter with a density of about a billion tons per cubic inch The American physicists R. Oppenheimer and H. Snyder, in 1939, realized that Schwarzschild theory was only possible if the star was much bigger than our sun; approximately 3.9 times or larger. In the early seventies, an $\mathrm{x}$-ray source was located in the Constellation Cygnus. The $\mathrm{x}$-ray source couldn't actually be precisely located but was narrowed down to a relatively small area. At this time it was very puzzling for the scientist. Steven Hawking, in the seventies, came up with theoretical arguments demonstrating that black holes aren't entirely black and that it is possible for them to evaporate because they emit radiation. The radiation from a black hole comes from the mass of it, hence the black hole insinuatingly shrinks. While the radiation tediously increases, the mass slowly decreases. So as the radiation from the black hole increases the black hole shrinks and evidently vanishes. In 1972, scientist pinpointed a radio emission in the same area. This is still puzzling to scientist today but all they knew was that it was originated from the same area as the $\mathrm{x}$-ray source and apparently came from open space. The scientist watched this area for months and finally waited until the spot in space came over the star next to it. When it did there was startling results. The spot in space eclipsed the star next to it. The spot was then named Cygnus X-1 and was officially the first Black hole discovered. Even back in Isaac Newton's time, scientist speculated such an object existed. So many questions had been answered by the discovery of this mysterious object, yet so many more generated.
Table of Time line of the discovery of black hole

\begin{tabular}{|c|c|c|}
\hline 1687 & Sir Isaac Newton & $\begin{array}{l}\text { Described gravity in his } \\
\text { publication, "Principia." }\end{array}$ \\
\hline 1783 & John Michell & $\begin{array}{l}\text { Conjectured that there might } \\
\text { be an object massive enough to } \\
\text { have an escape velocity greater } \\
\text { than the speed of light. }\end{array}$ \\
\hline 1796 & Simon Pierre Laplace & $\begin{array}{l}\text { Predicted the existence of black } \\
\text { holes. "...[It] is therefore possible } \\
\text { that the largest luminous bodies } \\
\text { in the universe may, through } \\
\text { this cause, be invisible." -- Le } \\
\text { Système du Monde }\end{array}$ \\
\hline 1915 & Albert Einstein & $\begin{array}{l}\text { Published the Theory of General } \\
\text { Relativity, which predicted } \\
\text { space-time curvature. }\end{array}$ \\
\hline 1916 & Karl Schwarzschild & $\begin{array}{l}\text { Used Einstein's Theory of } \\
\text { General Relativity to define a } \\
\text { black hole. Defined gravitational } \\
\text { radius of black holes, later called } \\
\text { the Schwarzschild radius. }\end{array}$ \\
\hline 1926 & Sir Arthur Erdington & $\begin{array}{l}\text { Relativity expert who, along } \\
\text { with Einstein, opposed black } \\
\text { hole theory. }\end{array}$ \\
\hline 1935 & $\begin{array}{l}\text { S ubrahman y a } n \\
\text { Chandrasekhar }\end{array}$ & $\begin{array}{l}\text { Pioneer in theory of white } \\
\text { dwarfs that led to an } \\
\text { understanding of mass limits } \\
\text { that decide whether a star will } \\
\text { die as a white dwarf, neutron } \\
\text { star or black hole. }\end{array}$ \\
\hline 1964 & John Wheeler & Coined the term, "black hole." \\
\hline 1964 & Jocelyn Bell-Burnell & $\begin{array}{l}\text { Discovered neutron stars that, at } \\
\text { the time, were the densest matter } \\
\text { found through observations. }\end{array}$ \\
\hline 1970 & Stephen Hawking & $\begin{array}{l}\text { Defined modern theory of black } \\
\text { holes, which describes the final } \\
\text { fate of black holes. }\end{array}$ \\
\hline 1970 & Cygnus X - 1 & $\begin{array}{l}\text { The first good black hole } \\
\text { candidate that astronomers } \\
\text { found. It emits x-rays and has } \\
\text { a companion smaller than Earth } \\
\text { but with a mass greater than that } \\
\text { of a neutron star. }\end{array}$ \\
\hline 1994 & $\begin{array}{l}\text { Hubble } \\
\text { Telescope }\end{array}$ & $\begin{array}{l}\text { Provides best evidence to date } \\
\text { of super massive black holes } \\
\text { that lurk in the centre of some } \\
\text { galaxies. The Space Telescope } \\
\text { Imaging Spectrograph (STIS) } \\
\text { revealed large orbiting velocities } \\
\text { around the nucleus of these } \\
\text { galaxies, suggesting a huge mass } \\
\text { inside a very small region. }\end{array}$ \\
\hline
\end{tabular}

\section{Theory of Black Holes}

Though the concept of a black hole was first proposed in 1783 by John Michell, it was Albert Einstein's 
1915 theory of general relativity which put the idea on a firm theoretical footing. Einstein showed that gravity can bend the path of light just as it bends the path of any other moving object - the only reason we don't observe this effect in our daily lives is that light moves fast and gravity pulls weak. When this was confirmed by observations, the idea of a black hole became obvious. If we pack enough material together, its gravitational pull should be strong enough to not only bend light's path but also keep it from escaping, just as the Earth is strong enough to pull back much slower objects (like baseballs) to its surface.

\section{Formation of Black Holes}

Regular black holes are thought to form from heavy stars. When these stars end their lives in a supernova explosion, their cores collapse and gravity wins out over any other force that might be able to hold the star up.

Eventually, the star collapses so much that it is contained within its Schwarzschild radius, or event horizon, the boundary within which light cannot escape. At this point, the black hole is extremely tiny; a black hole with the mass of the Sun would fit in a small town, while one with the mass of the Earth would fit in the palm of your hand! The material inside the Schwarzschild radius will continue to collapse indefinitely, reaching the point where our understanding of the laws of physics breaks down. But no information from inside the Schwarzschild radius can escape to the outside world.

The intense black hole gravitation is strong enough to curve the time and space in its neighbourhood. The intense gravitation causes a kind of a funnel consequence in that space time. This causes matters to be attracted gravitationally to it. It is supposed that the matter's atoms that enter the black holes are crushed into smaller area. As more matters enter the black hole, the gravitational field becomes more massive and stronger. A black hole is what is left when a huge a star dies. The black hole core is very strong. It sucks anything to an extent that light cannot get away.

The famous black holes in space are stellar and supermassive black hole. Stellar black hole is created by the gravitational collapse of a huge star. The process is viewed as a supernova explosion. Supermassive black hole is the biggest black hole type in a galaxy, on the array of hundreds, thousands, to billions of solar masses. Most galaxies, Milky Way included, are believed to have, at their middle, Supermassive black holes.

Astronomers have recognized many stellar black hole candidates. They have also discovered supermassive black holes evidence at the middle of galaxies. In 1998, astronomers discovered compelling proof that a supermassive black hole that exceeds two million solar masses is situated near Sagittarius area in the middle of the Milky Way galaxy. The current results show that supermassive black hole exceeds four million solar masses.

Black holes sound like they're straight out of a science fiction story: objects so dense that nothing in the universe can escape from their gravitational pull. But over the past few decades astronomers have been steadily building up evidence that black holes are not only real, but, in fact, quite prevalent in the universe. It is now thought that almost all galaxies contain gigantic black holes in their centers, millions or even billions of times more massive than the Sun. Some of these beasts are among the most violent and energetic objects in the universe - active galactic nuclei and quasars, which shoot off jets even as they suck in surrounding gas - while others, often older ones like the black hole at the center of the Milky Way, are considerably more quiet feeders.

Galaxies are also thought to contain many examples of small black holes, with masses only a few times greater than that of the Sun. Astronomers have detected a handful of these in our galaxy, by observing the light emitted when they shred apart their companion star in a binary system. Several of these small black holes have been dubbed "micro quasars" because they produce miniature jets akin to those of their larger cousins

\section{Observing Black Holes: accretion disk}

Though the interior of the black hole is invisible, it can 
be viewed through its association with other objects. A black hole can be deduced by following the group of stars movement that orbits an area in a space. In other words, when gas enters stellar black hole from a companion star, the gas twists inwards, heating at a very high temperature and producing huge amounts of radiation that can be noticed from earthbound and earth-orbiting telescopes.

Locations that black holes can easily be found have been identified. One likely area is at the middle of galaxies. At this region the concentration of stars increases the possibility of larger stars to burn off their fuel, creating black holes which consumed each other until one huge black hole was created. Quasars, the bright area at the middle of galaxies that produces humongous quantities of energy, could possibly contain black holes.

We can't observe black holes directly, but we do see their effect on surrounding material - gas and dust which lets out its last gasp before being sucked into the black hole or flung away in a jet. Black holes, in fact, are extremely efficient at converting the energy of incoming material into emitted light. The gas which falls into a black hole doesn't plunge in directly, for the same reason the Earth doesn't plunge into the Sun. Instead, it tries to move around the black hole in an orbit, forming what is known as an accretion disk.

Material in the accretion disk slowly spirals inward as it loses energy due to friction - the huge gravitational tides near the black hole are excellent at ripping apart this material and heating it to high temperatures. The inner disks of super massive black holes reach thousands of degrees Kelvin (similar to the temperatures at the surface of a hot star), while smaller black holes can heat their disks to millions of degrees, where they emit in the x-ray part of the spectrum.

\section{Black hole radiation: Hawkings radiation}

Black holes are not entirely black but emit small amounts of thermal radiation, an effect that has become known as Hawking radiation. By applying quantum field theory to a static black hole background, Hawking determined that a black hole should emit particles in a perfect black body spectrum. The temperature of this thermal spectrum called Hawking temperature is proportional to the surface gravity of the black hole, which, for a Schwarzschild black hole, is inversely proportional to the mass. Hence, large black holes emit less radiation than small black holes.

A stellar black hole of one solar mass has a Hawking temperature of about 100 nanokelvins. This is far less than the $2.7 \mathrm{~K}$ temperature of the cosmic microwave background radiation. Stellar mass or larger black holes receive more mass from the cosmic microwave background than they emit through Hawking radiation and thus will grow instead of shrink. To have a Hawking temperature larger than $2.7 \mathrm{~K}$ (and be able to evaporate), a black hole needs to have less mass than the Moon. Such a black hole would have a diameter of less than a tenth of a millimeter.

If a black hole is very small the radiation effects are expected to become very strong. Even a black hole that is heavy compared to a human would evaporate in an instant. A black hole having weight equal to that of a car would have a diameter of about $10^{-24} \mathrm{~m}$ and take a nanosecond to evaporate, during which time it would briefly have a luminosity more than 200 times that of the sun. Lower mass black holes are expected to evaporate even faster; for example, a black hole of mass $1 \mathrm{TeV} /$ $c^{2}$ would take less than $10^{-88}$ seconds to evaporate completely. For such a small black hole, quantum gravitation effects are expected to play an important role and could even - although current developments in quantum gravity do not indicate so-hypothetically make such a small black hole stable

\section{Ergosphere}

The ergosphere is an oblate spheroid region outside of the event horizon, where objects cannot remain stationary. Rotating black holes are surrounded by a region of spacetime in which it is impossible to stand still, called the ergosphere. This is the result of a process known as frame-dragging; general relativity predicts that any rotating mass will tend to slightly "drag" along the spacetime immediately surrounding it. Any object near the rotating mass will tend to start moving in the direction of rotation. For a rotating black hole this effect becomes so strong near the event 
horizon that an object would have to move faster than the speed of light in the opposite direction to just stand still.

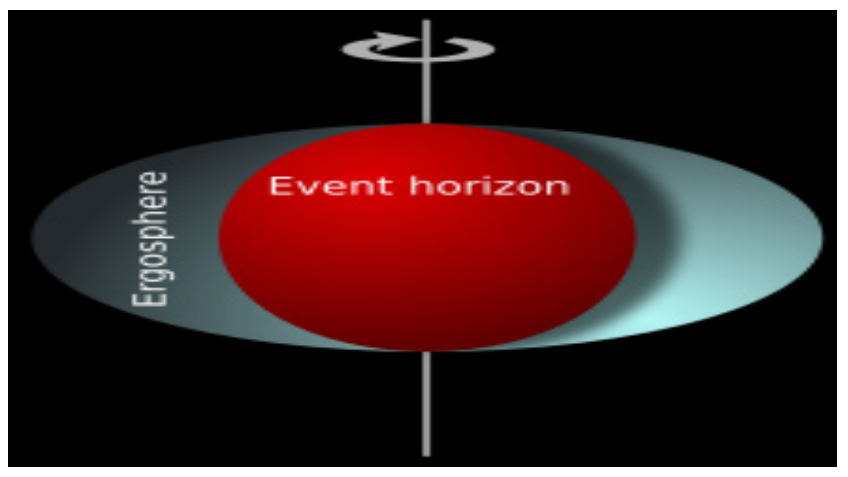

Fig 2 The ergosphere

The ergosphere of a black hole is bounded by the (outer) event horizon on the inside and an oblate spheroid, which coincides with the event horizon at the poles and is noticeably wider around the equator. The outer boundary is sometimes called the ergosurface. Objects and radiation can escape normally from the ergosphere. Through the Penrose process, objects can emerge from the ergosphere with more energy than they entered. This energy is taken from the rotational energy of the black hole causing it to slow down

\section{Fast variations}

The brightness of black holes can vary quickly. The complicated processes going on in the inner parts of the accretion disk are often highly variable, which leads to rapid changes in the amount of light being emitted. The smallest, most active black holes - the microquasars - can double their brightness in only a few seconds and show evidence for variability on much faster timescales, oscillating at hundreds of times per second in some cases.

\section{Energetic Jets}

Black holes suck material toward them, but some of it gets spit out rather than swallowed. Many black holes eject jets that move away from the accretion disk at nearly the speed of light. These energetic jets have been observed most spectacularly from the centers of nearby galaxies (for example, M87) but also appear in microquasars - in quick, enormously energetic spurts and sputters. The processes by which these jets are formed are not well understood, but seem to require magnetic fields - whose presence causes instabilities in the accretion disk that allow material to fling upwards as well as rapidly rotating black holes, which can feed some of their energy to the magnetic field and to the jet material itself.

\section{Solar System if the Sun suddenly become a black hole}

If the sun suddenly became a black hole, the planets would not be sucked into the black hole because their orbits would not change since the mass of the Sun has not changed. Only the space the Sun occupies has changed. The gravity of a black hole is very powerful only when we are very close to it. The only change is that it would be very dark and very cold.

\section{White Hole}

The equations of general theory of relativity have an interesting mathematical property: they are symmetric in time. That means that we can take any solution to the equations and imagine that time flows backward rather than forward, and we will get another valid solution to the equations. If we apply this rule to the solutions that describes black hole, we get an object known as a white hole. Since a black hole is a region of space from which nothing can escape, the time reversal version of a black hole is a region of space into which nothing can fall. In fact, just as a black hole can only suck things in, a white hole can only spit things out

A white hole is a theoretical space time region which cannot be accessed from outside. In a white hole, matter and light can easily escape. This is a reverse of a black hole. A black hole can be entered from outside but nothing including light can escape from it. White holes emerge in eternal black hole theory. In addition to a future black hole, Einstein equations solution has a white hole area in its past. However, this area is not available for black holes that have been created through gravitational collapse. There are no recognized physical procedures through which a white hole could be created.

Just like black holes, white holes comprise properties such as, mass, angular momentum, and charge. They 
attract object same as other mass. Objects that falls on a white hole cannot reach the event horizon of the white hole. However, according to maximally extended Schwarzschild solution, the event horizon of white hole in the past always becomes the event horizon of black hole in the future, therefore any object that falls towards the white hole will finally reach the event horizon of black hole.

In quantum mechanics, it is believed that black holes produces Hawking radiation, and it can reach thermal equilibrium with radiation's gas. Since a thermal stability state is instance reversal invariant, Hawking argued that black hole's time reverse in thermal stability is again a black hole in thermal stability. The Hawking radiation from a normal black hole is recognized with white hole production.

As a black hole irreversibly consumes anything that falls in it, a white hole only discharges energy and object out. However, since white holes seem to go against thermodynamics' second law it appears improbable that they exist in natural world. Albert Einstein discovered White holes. The holes seem as section of the vacuum explanation to the Einstein field equations explaining Schwarzschild wormhole. White holes emerge as a solution's part to the Einstein field equations. It explains an eternal black hole that does not have charge and rotation.

\section{Importance of Black Holes}

Indirectly black holes tell us that our relativistic theory of gravity and space-time provided by Einstein's general relativity is fundamentally correct. When we use these same equations to study cosmology we have some confidence that they may be correct and give us answers that make sense.

Directly, black holes tell us that the universe can hide much of its matter in a way that still contributes to the total mass of the universe, but may not contribute to the abundances of certain primordial elements such as hydrogen and helium. If enough black holes were produced soon after the big bang but before the first few minutes, this could have an impact on the relationship between how rapidly the universe is expanding and the origin of the primordial element abundances. But it is expected that most black holes formed long after the big bang by stellar evolution, and these black holes may contribute to the missing mass in the universe up to the maximum limit set by the primordial element abundances themselves. It is a bit complex, but black holes are cosmologically important from many different standpoints.

Black holes are an important phenomenon and may lead to new understanding of the nature of the "real universe". They are interesting objects from a fundamental physics point of view and play an important role in astrophysics processes. They help us to understand what's going on in the real universe

\section{Conclusion}

Black hole is a region of space-time formed by the gravitational collapse of massive star after the supernova explosion from which nothing, not even light can escape. As we have read, black holes are a major topic within our universe and they contain so much curiosity that they could possibly hold unlimited uses. Black holes are a sensation that astronomers are still very puzzled with. It seems that as we get closer to solving their existence and functions, we only end up with more and more questions. Although these questions just lead us into more and more unanswered problems we seek and find refuge into them, dreaming that maybe one far off/distant day, we will understand all the conceptions and we will be able to use the universe to our advantage and go where only our dreams could take us.

\section{References}

1. Alan Isaacs, Dictionary of Physics,1996 Oxford University Press New York

2. Arthur Beiser, Concept of Modern Physics, Fifth Edition, 1999, Mc GRAW Hill New York.

3. Poudel P., Gautam A., Adhikari B., Thapa M., Khatry M.,Principles of Physics for Class XI and XII,2010, Ayam Publication Kathmandu, Nepal

4. Microsoft corporation, Encarta, 2009, Microsoft corporation

5. Encyclopedia Britanica, 2010

6. Wikipedia, the free encyclopedia, en.wikipedia org/wiki. (Obtained; 2012.07) 